Published in final edited form as:

Bioconjug Chem. 2016 August 17; 27(8): 1789-1795. doi:10.1021/acs.bioconjchem.6b00235.

\title{
Pretargeted PET Imaging Using a Site-Specifically Labeled Immunoconjugate
}

\author{
Brendon E. Cook ${ }^{\dagger, \ddagger}$, , Pierre Adumeau ${ }^{\dagger}$, Rosemery Membreno ${ }^{\dagger, \ddagger}$, Kathryn E. Carnazza ${ }^{\S}$,

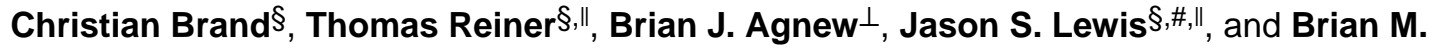 \\ Zeglis $\dagger, \neq, \S, \|, *$ \\ tDepartment of Chemistry, Hunter College of the City University of New York, 413 East 69th \\ Street, New York, New York 10028, United States \\ ¥Ph.D. Program in Chemistry, The Graduate Center of the City University of New York, 365 Fifth \\ Avenue, New York, New York 10016, United States \\ §Department of Radiology, Memorial Sloan Kettering Cancer Center, 1275 York Avenue, New \\ York, New York 10065, United States \\ \#Program in Molecular Pharmacology and Chemistry, Memorial Sloan Kettering Cancer Center, \\ 1275 York Avenue, New York, New York 10065, United States \\ "Department of Radiology, Weill Cornell Medical College, 520 East 70th Street, New York, New \\ York 10065, United States \\ ${ }^{\perp}$ Licensing and Commercial Supply, Thermo Fisher Scientific, 29851 Willow Creek Road, \\ Eugene, Oregon 97402, United States
}

\section{Abstract}

In recent years, both site-specific bioconjugation techniques and bioorthogonal pretargeting strategies have emerged as exciting technologies with the potential to improve the safety and efficacy of antibody-based nuclear imaging. In the work at hand, we have combined these two approaches to create a pretargeted PET imaging strategy based on the rapid and bioorthogonal inverse electron demand Diels-Alder reaction between a ${ }^{64} \mathrm{Cu}$-labeled tetrazine radioligand $\left({ }^{64} \mathrm{Cu}\right.$ Tz-SarAr) and a site-specifically modified huA33-trans-cyclooctene immunoconjugate ( ${ }^{\mathrm{SS}} \mathrm{huA33-}$ $\mathrm{PEG}_{12}-\mathrm{TCO}$ ). A bioconjugation strategy that harnesses enzymatic transformations and strainpromoted azide-alkyne click chemistry was used to site-specifically append PEGylated TCO moieties to the heavy chain glycans of the colorectal cancer-targeting huA33 antibody. Preclinical in vivo validation studies were performed in athymic nude mice bearing A33 antigen-expressing

\footnotetext{
*Corresponding Author. bz102@hunter.cuny.edu. Phone: 212-896-0443. Fax: 212-772-5332. ASSOCIATED CONTENT

Supporting Information

The Supporting Information is available free of charge on the ACS Publications website at DOI: 10.1021/acs.bioconjchem.6b00235.

Reagents and general procedures; detailed experimental methods for chemical syntheses, bioconjugation procedures, gel analyses, in vitro assays, and in vivo experiments; SDS-PAGE analyses of immunoconjugates before and after PNGaseF treatment; schematics of bioconjugation strategies; tables of biodistribution data; histology, immunofluorescence staining, and autoradiography of resected tissues; and tables of dosimetry data (PDF)
}

The authors declare no competing financial interest. 
SW1222 human colorectal carcinoma xenografts. To this end, mice were administered ${ }^{\text {ss }}$ huA33$\mathrm{PEG}_{12}$-TCO via tail vein injection and-following accumulation intervals of 24 or $48 \mathrm{~h}-{ }^{64} \mathrm{Cu}$ Tz-SarAr. PET imaging and biodistribution studies reveal that this strategy clearly delineates tumor tissue as early as $1 \mathrm{~h}$ post-injection $(6.7 \pm 1.7 \% \mathrm{ID} / \mathrm{g}$ at $1 \mathrm{~h}$ p.i.), producing images with excellent contrast and high tumor-to-background activity concentration ratios (tumor: muscle $=$ $21.5 \pm 5.6$ at $24 \mathrm{~h}$ p.i.). Furthermore, dosimetric calculations illustrate that this pretargeting approach produces only a fraction of the overall effective dose $(0.0214 \mathrm{mSv} / \mathrm{MBq} ; 0.079 \mathrm{rem} / \mathrm{mCi})$ of directly labeled radioimmunoconjugates. Ultimately, this method effectively facilitates the high contrast pretargeted PET imaging of colorectal carcinoma using a site-specifically modified immunoconjugate.

\section{Graphical Abstract}

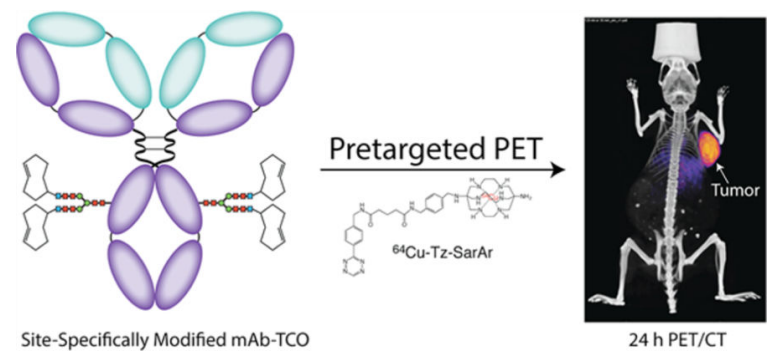

\section{INTRODUCTION}

The use of radiolabeled antibodies for the noninvasive imaging of cancer biomarkers stands poised to be a vital component in the emerging era of personalized medicine. ${ }^{1}$ Yet despite this promise, a number of important obstacles stand in the way of the widespread clinical implementation of radioimmunoconjugates. Indeed, from a bioconjugation standpoint, two especially troublesome issues stand out. First, the relatively long biological half-life of antibodies mandates the use of radioisotopes with comparable physical half-lives, for example ${ }^{89} \mathrm{Zr}\left(t_{1 / 2} \sim 3.2 \mathrm{~d}\right)$ or ${ }^{124} \mathrm{I}\left(t_{1 / 2} \sim 4.2 \mathrm{~d}\right) .{ }^{2-4}$ Not surprisingly, this exigency can lead to high radiation doses to the healthy organs of the patient. ${ }^{5-11}$ Second, traditional methods of antibody modification rely on the covalent coupling of amine-reactive probes to the lysine residues of the antibody. However, due to the presence of multiple lysines on the surface of immunoglobulins, controlling the location and frequency of these couplings is impossible. This non-site-specific approach to bioconjugation can yield poorly defined and heterogeneous immunoconjugates that suffer from suboptimal in vivo behavior, specifically decreased uptake in target tissues and increased retention in healthy organs. ${ }^{12-18}$

In recent years, significant effort from an array of research groups has been dedicated to addressing these concerns through the development of novel approaches to pretargeted imaging and site-specific bioconjugation. ${ }^{12,14-17}$ Our laboratories have contributed two different methodologies based on bioorthogonal chemistry. ${ }^{19-22}$ First, in order to counter the dosimetric limitations of traditional radioimmunoconjugates, we have created a pretargeted PET imaging approach that leverages the extraordinarily rapid inverse electron-demand Diels-Alder cycloaddition between tetrazine (Tz) and trans-cyclooctene (TCO) (Figure 
1A). ${ }^{23-25}$ This method has four straightforward steps: (1) the injection of a TCO-modified antibody; (2) the accumulation of the TCO-labeled antibody at the tumor and its simultaneous clearance from the blood; (3) the injection of a small molecule Tz-bearing radioligand; and (4) the in vivo click ligation between the two components followed by the excretion of any excess radioligand (Figure 2). Importantly, in murine models of both colorectal and pancreatic cancer, we have shown that this strategy delineates tumor tissue with excellent image contrast at only a small fraction of the radiation dose to healthy tissues created by traditional immunoconjugates. ${ }^{21,22,26}$

We turned to a second bioorthogonal reaction - the strain-promoted azide-alkyne cycloaddition (SPAAC) — in our effort to improve upon the site-specificity of traditional bioconjugation techniques (Figure 1B). ${ }^{23,27}$ We developed a robust and modular chemoenzymatic strategy for the site-specific modification of antibodies on the heavy chain glycans, the conserved biantennary oligosaccharide chains located on the $\mathrm{C}_{\mathrm{H}} 2$ regions of immunoglobulins. ${ }^{19,28}$ This approach relies on the enzymatic activity of $\beta$-1,4-galactosidase and a promiscuous galactosyltransferase [Gal-T(Y289L)] for the incorporation of azidemodified monosaccharides - GalNAz — into the sugar chains. The resulting azide-modified antibody can then be easily conjugated to any dibenzocyclooctyne-bearing cargo via the SPAAC ligation. In three different murine model systems, we have illustrated that this approach produces well-defined and homogeneous immunoconjugates with in vivo behavior comparable - and often superior - to immunoconjugates synthesized using traditional, nonsite-specific methods. ${ }^{26,28,29}$

In the work at hand, we have successfully combined these two methodologies to create a pretargeted PET imaging strategy using a ${ }^{64} \mathrm{Cu}$-labeled tetrazine radioligand $\left({ }^{64} \mathrm{Cu}-\mathrm{Tz}-\right.$ SarAr) and a site-specifically modified antibody-TCO immunoconjugate (Figure 2).

The driving force behind this investigation lies in our sincere belief that the use of a sitespecifically modified immunoconjugate could improve our pretargeting strategy in two ways. First, a site-specifically modified huA33-TCO construct would be far better defined and much more homogeneous than its randomly labeled cousin, a distinction that we believe could prove vital during the regulatory approval process. Second, a wide array of evidence has suggested that site-specifically modified immunoconjugates exhibit significantly improved in vivo behavior compared to analogous constructs synthesized using random labeling methods. ${ }^{30-35}$ Pillow et al., for example, recently found that a site-specifically labeled trastuzumab-maytansinoid antibody-drug conjugate (ADC) displayed improved therapeutic activity in vivo compared to an analogous non-site-specifically labeled ADC. ${ }^{31}$ As a result, we believe that a site-specifically labeled huA33-TCO immunoconjugate could improve the tumoral activity concentrations produced by the system and further reduce the already very low activity concentrations in nontarget healthy tissues. Ultimately, we have shown that this approach enables the rapid and clear visualization of tumor tissue with excellent tumor-to-background activity concentration ratios at organ-specific and overall effective radiation doses far below those created by non-pretargeted methodologies. 


\section{RESULTS AND DISCUSSION}

The starting point for this investigation was the optimized pretargeted PET imaging methodology recently reported by our laboratory. ${ }^{22,26}$ This system employs two components: a ${ }^{64} \mathrm{Cu}$-labeled tetrazine radioligand $\left({ }^{64} \mathrm{Cu}-\mathrm{Tz}\right.$-SarAr) and a trans-cyclooctenebearing immunoconjugate of the huA33 antibody (huA33-TCO) (Figure 2B). The huA33 antibody targets the A33 antigen, a transmembrane glycoprotein that is expressed by $>95 \%$ of colorectal carcinomas and, critically, is not internalized or shed upon forming an immunocomplex with the antibody. ${ }^{36-42}$ This imaging approach clearly delineates antigenexpressing tumor tissue with high tumor-to-background activity concentration ratios and very low radiation doses to healthy tissues when compared to traditional, directly labeled radioimmunoconjugates. ${ }^{22}$ However, we contend that there is room for improvement, specifically by employing a more homogeneous and well-defined TCO-modified immunoconjugate synthesized via site-specific bioconjugation.

The site-specific modification of huA33 was performed using a three-step process that leverages both enzymatic transformations and a second variety of bioorthogonal click chemistry (Figure 2A). First, the terminal galactose residues on the heavy chain glycans were removed via incubation with $\beta$-1,4-galactosidase. Next, Gal-T(Y289L), a promiscuous galactosyltransferase, was used to attach azide-modified sugars (GalNAz) to the termini of the polysaccharide chains to produce an huA33- $\mathrm{N}_{3}$ immunoconjugate with $3.4 \pm 0.3 \mathrm{~N}_{3} /$ $\mathrm{mAb} .{ }^{43-49}$ Finally, the azide-modified antibody was conjugated to a dibenzocyclooctynebearing variant of TCO (DIBO-PEG 12 -TCO) via the strain-promoted azide-alkyne cycloaddition to give the final product: ${ }^{\text {ss }}$ huA33- $\mathrm{PEG}_{12}-\mathrm{TCO}$.

The characterization of the ${ }^{\mathrm{ss}} \mathrm{huA} 33-\mathrm{PEG}_{12}-\mathrm{TCO}$ was carried out to interrogate both the number and the placement of the $\mathrm{PEG}_{12}$-TCO moieties on the antibody. MALDI-TOF mass spectrometry analysis of ${ }^{\mathrm{Ss}} \mathrm{huA} 33-\mathrm{PEG}_{12}-\mathrm{TCO}$ revealed that the immunoconjugate was modified with $2.4 \pm 0.5 \mathrm{TCO} / \mathrm{mAb}$. Of course, this result means that the final ${ }^{\mathrm{ss}} \mathrm{huA} 33$ $\mathrm{PEG}_{12}$-TCO immunoconjugate contains $\sim 1$ unmodified terminal GalNAz; however, given that azides do not react with tetrazines, it is extraordinarily unlikely that the presence of the $\mathrm{N}_{3}$ functionality would interfere with the in vivo click ligation. Next, the site-specificity of the modification was confirmed via denaturing SDS-PAGE analysis (see Supporting Information Figure S1). In these experiments, a positive shift in the molecular weight of the heavy chain was observed for ${ }^{\text {ss }}$ huA33-PEG 12 -TCO compared to that of unmodified huA33, while no molecular weight difference was observed between the light chains of the two constructs. The SDS-PAGE analysis of immunoconjugates that had been treated with PNGaseF-an amidase that removes the heavy chain glycans entirely-provided additional insight (see Supporting Information Figure S2). In these assays, a shift in molecular weight of the heavy chain could be observed in samples that had not been treated with PNGaseF, while no difference was observed in samples that had been treated with the enzyme. Taken together, these electrophoresis experiments clearly illustrate that the bioconjugation of TCO is not only specific to the heavy chain of the antibody but is specific to the heavy chain glycans of the antibody. 
As a final characterization step prior to in vivo experiments, the in vitro immunoreactivity of ${ }^{\text {ss }}$ huA33-PEG $12-\mathrm{TCO}$ was measured. To this end, ${ }^{\text {ss }}$ huA33-PEG $12-\mathrm{TCO}$ was radiolabeled via click ligation with ${ }^{64} \mathrm{Cu}-\mathrm{Tz}-\mathrm{SarAr}$, purified via size exclusion chromatography, and incubated with a vast excess of A33 antigen-expressing SW1222 human colorectal carcinoma cells. Using this antigen saturation technique, it was determined that the ${ }^{64} \mathrm{Cu}$ labeled immunoconjugate maintained a very high immunoreactive fraction of $0.94 \pm 0.02$, an unsurprising result considering that our site-specific modification occurs on the $\mathrm{C}_{\mathrm{H}} 2$ domains of the $\mathrm{Fc}$ region, far from the antigen-binding domains of the immunoglobulin.

Once fully characterized, the ${ }^{\mathrm{s}}$ huA33- $\mathrm{PEG}_{12}$ - $\mathrm{TCO}$ immunoconjugate was ready to be put to the test in vivo. To this end, athymic nude mice bearing subcutaneous A33 antigenexpressing SW1222 human colorectal carcinoma xenografts $\left(5 \times 10^{6}\right.$ cells; right shoulder; 16-18 days post-inoculation) were used as a model system. Informed by our previous efforts toward the optimization of this pretargeting approach, these mice were first injected with ${ }^{\mathrm{ss}} \mathrm{huA} 33-\mathrm{PEG}_{12}-\mathrm{TCO}(0.66 \mathrm{nmol})$ and then-following a $24 \mathrm{~h}$ accumulation interval - ${ }^{64} \mathrm{Cu}-\mathrm{Tz}-\operatorname{SarAr}(300-350 \mu \mathrm{Ci} ; 11.1-13.0 \mathrm{MBq}$; 0.66-0.77 nmol). Static PET scans were subsequently acquired 4,12 , and $24 \mathrm{~h}$ after the administration of the radiotracer (Figure 3 ). These images clearly indicate that this pretargeting approach quickly and effectively visualizes the antigen-expressing tumor tissue. As early as $4 \mathrm{~h}$ post-injection, the tumor tissue is clearly delineated. Critically, image contrast improves with time as the excess radioligand is cleared via the urinary tract. Indeed, by $24 \mathrm{~h}$ p.i. the only tissue displaying appreciable uptake of the radiotracer is the tumor, with only minimal background signal remaining in healthy organs.

An acute biodistribution experiment was performed to more accurately quantify the in vivo behavior of the pretargeting system (Figure 4). The biodistribution was performed under conditions identical to the PET experiment and largely corroborated the imaging results. Significant tumor uptake can be observed in the first hour post-injection $(6.7 \pm 1.7 \% \mathrm{ID} / \mathrm{g})$ and increases over the course of the experiment, ultimately reaching $11.2 \pm 2.4 \% \mathrm{ID} / \mathrm{g}$ at $24 \mathrm{~h}$ p.i. Conversely, the activity concentration in the blood pool followed an opposite trajectory, starting at $9.5 \pm 1.6 \% \mathrm{ID} / \mathrm{g}$ at $1 \mathrm{~h}$ p.i. and decreasing until it reached $4.0 \pm 0.7 \% \mathrm{ID} / \mathrm{g}$ at $24 \mathrm{~h}$ p.i. This trend of clearance is mirrored - though at significantly lower activity concentration levels-in nearly all other organs sampled, including the heart, lungs, liver, and spleen. The kidneys demonstrated the highest nontarget uptake $(2.7 \pm 0.1 \% \mathrm{ID} / \mathrm{g}$ at $24 \mathrm{~h}$ p.i.), an observation consistent with our previous findings. ${ }^{21}$ Two critical metrics for evaluating the efficacy of ${ }^{\mathrm{ss}} \mathrm{huA} 33-\mathrm{PEG}_{12}-\mathrm{TCO}$ are the tumor-to-large intestine and tumor-to-liver activity concentration ratios, as these organs represent the most clinically relevant background tissues for primary and metastatic disease, respectively. The tumor-to-large intestine ratio was exceptional $(18.0 \pm 5.2$ at $24 \mathrm{~h}$ p.i.), while the tumor-to-liver ratio was lower $(5.2 \pm 1.4$ at $24 \mathrm{~h}$ p.i.) but still quite promising.

In an effort to decrease the residual levels of activity in the blood, an additional pretargeted biodistribution study was performed using a longer accumulation interval of $48 \mathrm{~h}$ (see Supporting Information Figure S3). As expected, the use of a prolonged accumulation interval decreased activity concentrations in the blood, a result that almost certainly stems from reducing the amount of ${ }^{\mathrm{s}} \mathrm{huA} 33-\mathrm{PEG}_{12}-\mathrm{TCO}$ remaining in the blood at the time of the 
injection of the radioligand and thus concomitantly decreasing the amount of ${ }^{64} \mathrm{Cu}$-labeled huA33 that is formed through click ligations within the blood pool. Unfortunately, however, the use of a $48 \mathrm{~h}$ accumulation interval also resulted in small reductions to the amount of uptake in the tumor, a phenomenon possibly related to either the slow in vivo isomerization of reactive TCO to unreactive cis-cyclooctene, the in vivo stability of the immunoconjugate, or a combination thereof. Clearly, as in all pretargeting approaches, the accumulation interval must be carefully tailored to strike the critical yet delicate balance between the activity concentrations in the tumor and healthy organs. Finally, it is important to note that in vivo experiments performed in previous investigations using ${ }^{64} \mathrm{Cu}-\mathrm{Tz}-\mathrm{SarAr}$ alone, pretargeting with a nonspecific isotype control $\mathrm{IgG}$, or pretargeting with an unmodified huA33 construct have been shown to yield minimal $(<1.5 \%$ ID/g) tumoral activity concentrations. $^{26}$

Importantly, control biodistribution studies were performed using huA33 non-sitespecifically labeled with TCO-NHS ( ${ }^{\text {ns }}$ huA33-TCO) and huA33 non-site-specifically labeled with TCO-PEG 12 -NHS (nshuA33-PEG 12 -TCO) (see Supporting Information Figure S4). To this end, athymic nude mice bearing SW1222 xenografts were injected

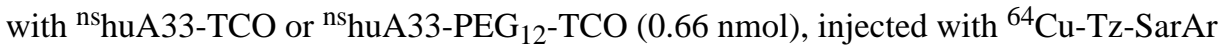
(300-350 $\mu \mathrm{Ci}$; 11.1-13.0 MBq; 0.66-0.77 nmol) after an accumulation interval of $24 \mathrm{~h}$, and sacrificed either 4 or $24 \mathrm{~h}$ after the administration of the radioligand (see Supporting Information Figure S5). Importantly, the tumor and healthy organ activity concentrations obtained with all three immunoconjugates were very similar. Interestingly, however, the activity concentration remaining in the blood using ${ }^{\mathrm{ss}} \mathrm{huA} 33-\mathrm{PEG}_{12}$-TCO $(4.0 \pm 0.7 \% \mathrm{ID} / \mathrm{g}$ at $24 \mathrm{~h}$ p.i. $)$ was higher than that measured using ${ }^{\mathrm{ns}} \mathrm{huA} 33-\mathrm{TCO}(2.4 \pm 0.8 \% \mathrm{ID} / \mathrm{g}$ at $24 \mathrm{~h}$ p.i. $)$ or ${ }^{\text {ns }}$ huA33-PEG 12 -TCO (3.1 $\pm 0.5 \%$ ID/g at $24 \mathrm{~h}$ p.i.). While the origins of this phenomenon are unclear, it may stem from an increase in the blood-half-life of sshuA33-PEG 12 -TCO related to the presence of the bulky DIBO and PEG $_{12}$ moieties (see DIBO structure in Figure 1). On the whole, however, these data underscore that pretargeted PET imaging using a more homogeneous and better defined site-specifically modified immunoconjugate is possible without significantly sacrificing in vivo performance.

Moving on, it was important to verify that the in vivo click reaction-and not simply the nonspecific localization of ${ }^{64} \mathrm{Cu}-\mathrm{Tz}-\mathrm{SarAr}$ - was responsible for the strategy's effective delineation of tumor tissue. To this end, at the conclusion of the imaging studies, representative tumors were resected, and slices were made for autoradiographic and immunohistological analysis (see Supporting Information Figure S6). Comparison of the hematoxylin and eosin (H\&E) stain with the immunofluorescence staining for huA33 clearly shows that the huA33 construct associates only with tumor cells and not vasculature, stroma, or areas of necrosis. Perhaps even more importantly, there is significant overlap between areas of high huA33 density and increased activity in the autoradiograph image, illustrating the colocalization of ${ }^{64} \mathrm{Cu}$ and huA33. This clearly shows that we have achieved the successful in vivo ligation of the radioligand to the modified antibody. Finally, the localization of huA33 can also be seen to occur on the outside of the cells, further corroborating the surface persistence of the huA33-A33 antigen immunocomplex. ${ }^{26}$ 
Finally, the biodistribution data obtained with ${ }^{64} \mathrm{Cu}-\mathrm{Tz}-\mathrm{SarAr}$ and ${ }^{\mathrm{ss}} \mathrm{huA} 33-\mathrm{PEG}_{12}-\mathrm{TCO}$ were used to perform dosimetry calculations for the pretargeted PET imaging approach. To this end, activity curves were constructed using results from the biodistribution data, and then integrated to provide time-activity data for each organ. The OLINDA computer program was then used to determine the mean organ absorbed doses and effective dose for the methodology (see Supporting Information Table S1). The effective dose for pretargeting with ${ }^{64} \mathrm{Cu}$-Tz-SarAr and ${ }^{\text {ss }}$ huA33-PEG 12 -TCO was $0.0214 \mathrm{mSv} / \mathrm{MBq}(0.079 \mathrm{rem} / \mathrm{mCi})$, only slightly higher than that of the nonsite-specific pretargeting approach $(0.012 \mathrm{mSv} / \mathrm{MBq}$; $0.041 \mathrm{rem} / \mathrm{mCi}$ ) previously reported. ${ }^{22}$ The higher absorbed dose for this site-specific system is most likely due to the addition of the bulky DIBO moieties and hydrophilic PEG $_{12}$ chains, which can increase circulation times and thus increase uptake and retention in healthy organs. Finally, the effective dose of ${ }^{\mathrm{Ss}} \mathrm{huA} 33-\mathrm{PEG}_{12}$-TCO is nearly 20 -times lower than that produced by its most likely clinical alternative: directly labeled ${ }^{89} \mathrm{Zr}$-DFO-huA33 $(0.416 \mathrm{mSv} / \mathrm{MBq} ; 1.54 \mathrm{rem} / \mathrm{mCi}){ }^{26}$

\section{CONCLUSION}

In the preceding pages, we have reported the development of a site-specifically labeled huA33-TCO immunoconjugate for the pretargeted PET imaging of colorectal cancer. Our approach quickly and clearly delineates tumors with both high tumor-to-healthy tissue activity concentration ratios and low dose rates to healthy tissues. Admittedly, however, it seems that we have hit the mark emphatically on only one of our two original goals. The site-specifically modified huA33-PEG 12 - $\mathrm{TCO}$ immunoconjugate described in these pages is unquestionably better defined and more homogeneous than the huA33-TCO constructs reported in our previous work. Yet, while the performance of the pretargeted PET imaging methodology using huA33- $\mathrm{PEG}_{12}-\mathrm{TCO}$ is comparable to pretargeting using a randomly labeled variant of huA33-TCO, we cannot necessarily argue that it is superior. In the end, then, this study has illustrated that it is possible to perform pretargeted PET imaging using a site-specific bioconjugation approach without sacrificing in vivo performance. Furthermore, it is important to note that both the bioorthogonal pretargeting strategy and the site-specific modification approach are highly modular: both techniques can be applied to any tumortargeting immunoglobulin for a wide variety of applications. Ultimately, our approach facilitates pretargeted PET imaging with a well-defined and homogeneous immunoconjugate, an advance that will hopefully help expedite the clinical translation of future pretargeting strategies.

\section{Supplementary Material}

Refer to Web version on PubMed Central for supplementary material.

\section{Acknowledgments}

Services provided by the MSKCC Small-Animal Imaging Core Facility were supported in part by NIH grants R24 CA83084 and P30 CA08748. The authors are also grateful for the generous financial support of the National Institutes of Health (4R00CA178205-02; BMZ), the Team Connor Childhood Cancer Foundation (BMZ), the National Institute on Minority Health and Health Disparities (G12MD007599; BMZ), the Tow Foundation Fellowship Program in Molecular Imaging and Nanotechnology (JSL; BEC), and Mr. William H. Goodwin and 
Mrs. Alice Goodwin and the Commonwealth Foundation for Cancer Research and The Center for Experimental Therapeutics at Memorial Sloan Kettering Cancer Center (JSL).

\section{REFERENCES}

1. Wu AM. Antibodies and Antimatter: The Resurgence of Immuno-PET. J. Nucl. Med. 2008; 50:2-5. [PubMed: 19091888]

2. Deri MA, Zeglis BM, Francesconi LC, Lewis JS. PET imaging with 89Zr: From radiochemistry to the clinic. Nucl. Med. Biol. 2013; 40:3-14. [PubMed: 22998840]

3. van Dongen GAMS, Visser GWM, Lub-de Hooge MN, de Vries EG, Perk LR. Immuno-PET: a navigator in monoclonal antibody development and applications. Oncologist. 2007; 12:1379-1389. [PubMed: 18165614]

4. Vugts DJ, van Dongen GAMS. 89Zr-labeled compounds for PET imaging guided personalized therapy. Drug Discovery Today: Technol. 2011; 8:e53-e61.

5. Sharkey RM, Goldenberg DM. Cancer radioimmunotherapy. Immunotherapy. 2011; 3:349-370. [PubMed: 21395378]

6. Sharkey RM, Karacay H, Cardillo TM, Chang CH, McBride WJ, Rossi EA, Horak ID, Goldenberg DM. Improving the delivery of radionuclides for imaging and therapy of cancer using pretargeting methods. Clin. Cancer Res. 2005; 11:7109-7121.

7. Sharkey RM, Chang CH, Rossi EA, McBride WJ, Goldenberg DM. Pretargeting: Taking an alternate route for localizing radionuclides. Tumor Biol. 2012; 33:591-600.

8. Goldenberg DM, Sharkey RM, Paganelli G, Barbet J, Chatal JF. Antibody pretargeting advances cancer radioimmunodetection and radioimmunotherapy. J. Clin. Oncol. 2006; 24:823-834. [PubMed: 16380412]

9. Goldenberg DM, Rossi EA, Sharkey RM, McBride WJ, Chang CH. Multifunctional antibodies by the dock-and-lock method for improved cancer Imaging and therapy by pretargeting. J. Nucl. Med. 2007; 49:158-163. [PubMed: 18077530]

10. Goldenberg DM, Chang CH, Rossi EA, McBride WJ, Sharkey RM. Pretargeted molecular imaging and radioimmunotherapy. Theranostics. 2012; 2:523-540. [PubMed: 22737190]

11. Boerman OC, van Schaijk FG, Oyen WJG, Corstens FHM. Pretargeted radioimmunotherapy of cancer: Progress step by step. J. Nucl. Med. 2003; 44:400-411. [PubMed: 12621007]

12. Agarwal P, Bertozzi CR. Site-specific antibody-drug conjugates: the nexus of bioorthogonal chemistry, protein engineering, and drug development. Bioconjugate Chem. 2015; 26:176-192.

13. Adumeau P, Sharma SK, Brent C, Zeglis BM. Site-Specifically Labeled Immunoconjugates for Molecular Imaging_Part 1: Cysteine Residues and Glycans. Mol. Imaging Biol. 2016; 18:1-17.

14. Jeger S, Zimmermann K, Blanc A, Grunberg J, Honer M, Hunziker P, Struthers H, Schibli R. Sitespecific and stoichiometric modification of antibodies by bacterial transglutaminase. Angew. Chem., Int. Ed. 2010; 49:9995-9997.

15. Rossin R, Verkerk PR, van den Bosch SM, Vulders RCM, Verel I, Lub J, Robillard MS. In vivo chemistry for pretargeted tumor imaging in live mice. Angew. Chem., Int. Ed. 2010; 49:33753378.

16. Rossin R, Lappchen R, van den Bosch SM, LaForest R, Robillard MS. Diels-Alder reaction for tumor pretargeting: In vivo chemistry can boost tumor radiation dose compared with directly labeled antibody. J. Nucl. Med. 2013; 54:1989-1995. [PubMed: 24092936]

17. Devaraj NK, Thurber GM, Keliher EJ, Marinelli B, Weissleder R. Reactive polymer enables efficient in vivo bioorthogonal chemistry. Proc. Natl. Acad. Sci. U. S. A. 2012; 109:4762-4767. [PubMed: 22411831]

18. Zhou Q, Stefano JE, Manning C, Kyazike J, Chen B, Gianolio DA, Park A, Busch M, Bird J, Zheng X, et al. Site-specific antibody-drug conjugation through glycoengineering. Bioconjugate Chem. 2014; 25:510-520.

19. Zeglis BM, Davis CB, Aggeler R, Kang HC, Chen A, Agnew BJ, Lewis JS. An enzyme-mediated methodology for the site-specific radiolabeling of antibodies based on catalyst-free click chemistry. Bioconjugate Chem. 2013; 24:1057-1067. 
20. Zeglis BM, Davis CB, Abdel-Atti D, Carlin SD, Chen A, Aggeler R, Agnew BJ, Lewis JS. Chemoenzymatic strategy for the synthesis of site-specifically labeled immunoconjugates for multimodal PET and optical imaging. Bioconjugate Chem. 2014; 25:2123-2128.

21. Meyer JP, Houghton JL, Kozlowski P, Abdel-Atti D, Reiner T, Pillarsetty NV, Scholz WW, Zeglis BM, Lewis JS. ${ }^{18}$ F-Based Pretargeted PET Imaging Based on Bioorthogonal Diels-Alder Click Chemistry. Bioconjugate Chem. 2016; 27:298-301.

22. Zeglis BM, Brand C, Abdel-Atti D, Carnazza KE, Cook BE, Carlin S, Reiner T, Lewis JS. Optimization of a Pretargeted Strategy for the PET Imaging of Colorectal Carcinoma via the Modulation of Radioligand Pharmacokinetics. Mol. Pharmaceutics. 2015; 12:3575-3587.

23. Reiner T, Zeglis BM. The inverse electron demand Diels-Alder click reaction in radiochemistry. J. Labelled Compd. Radiopharm. 2014; 57:285-290.

24. Devaraj NK, Weissleder R. Biomedical applications of tetrazine cycloadditions. Acc. Chem. Res. 2011; 44:816-827. [PubMed: 21627112]

25. Seitchik JL, Peeler JC, Taylor MT, Blackman ML, Rhoads TW, Cooley RB, Refakis C, Fox JM, Mehl RA. Genetically encoded tetrazine amino acid directs rapid site-specific in vivo bioorthogonal ligation with trans-cyclooctenes. J. Am. Chem. Soc. 2012; 134:2898-2901. [PubMed: 22283158]

26. Zeglis BM, Sevak KK, Reiner T, Mohindra P, Carlin SD, Zanzonico P, Weissleder R, Lewis JS. A pretargeted PET imaging strategy based on bioorthogonal Diels-Alder click chemistry. J. Nucl. Med. 2013; 54:1389-1396. [PubMed: 23708196]

27. Sletten EM, Bertozzi CR. Bioorthogonal chemistry: fishing for selectivity in a sea of functionality. Angew. Chem., Int. Ed. 2009; 48:6974-6998.

28. Zeglis BM, Davis CB, Abdel-Atti D, Carlin SD, Chen A, Aggeler R, Agnew BJ, Lewis JS. Chemoenzymatic strategy for the synthesis of site-specifically labeled immunoconjugates for multimodal PET and optical imaging. Bioconjugate Chem. 2014; 25:2123-2128.

29. Houghton JL, Zeglis BM, Abdel-Atti D, Aggeler R, Sawada R, Agnew BJ, Scholz WW, Lewis JS. Site-specifically labeled CA19.9-targeted immunoconjugates for the PET, NIRF, and multimodal PET/NIRF imaging of pancreatic cancer. Proc. Natl. Acad. Sci. U. S. A. 2015; 112:15850-15855. [PubMed: 26668398]

30. Junutula JR, Raab H, Clark S, Bhakta S, Leipold DD, Weir S, Chen Y, Simpson M, Tsai SP, Dennis MS, et al. Site-specific conjugation of a cytotoxic drug to an antibody improves the therapeutic index. Nat. Biotechnol. 2008; 26:925-932. [PubMed: 18641636]

31. Pillow TH, Tien J, Parsons-Reponte KL, Bhakta S, Li H, Staben LR, Li G, Chuh J, FourieO’Donohue A, Darwish M, et al. Site-Specific Trastuzumab Maytansinoid Antibody-Drug Conjugates with Improved Therapeutic Activity through Linker and Antibody Engineering. J. Med. Chem. 2014; 57:7890-7899. [PubMed: 25191794]

32. Boswell CA, Marik J, Elowson MJ, Reyes NA, Ulufatu S, Bumbaca D, Yip V, Mundo EE, Majidy $\mathrm{N}$, Van Hoy M, et al. Enhanced tumor retention of a radiohalogen label for site-specific modification of antibodies. J. Med. Chem. 2013; 56:9418-9426. [PubMed: 24131491]

33. Boswell CA, Mundo EE, Zhang C, Bumbaca D, Valle NR, Kozak KR, Fourie A, Chuh J, Koppada $\mathrm{N}$, Saad O, et al. Impact of drug conjugation on pharmacokinetics and tissue distribution of antiSTEAP1 antibody-drug conjugates in rats. Bioconjugate Chem. 2011; 22:1994-2004.

34. Alvarez VL, Wen ML, Lee C, Lopes AD, Rodwell JD, McKearn TJ. Site-specifically modified In-111 labeled antibodies give low liver backgrounds and improved radioimmunoscintigraphy. Nucl. Med. Biol. 1986; 13:347-352.

35. Strop P, Liu S-H, Dorywalska M, Delaria K, Dushin RG, Tran T-T, Ho W-H, Farias S, Casas MG, Abdiche Y, et al. Location matters: Site of conjugation modulates stability and pharmacokinetics of antibody drug conjugates. Chem. Biol. 2013; 20:161-167. [PubMed: 23438745]

36. Schoffelen R, Sharkey RM, Goldenberg DM, Franssen G, McBride WJ, Rossi EA, Chang CH, Laverman P, Disselhorst JA, Eek A, et al. Pretargeted immuno-positron emission tomography imaging of carcinoembryonic antigen-expressing tumors with a bispecific antibody and a $68 \mathrm{Ga}-$ and $18 \mathrm{~F}$-labeled hapten peptide in mice with human tumor xenografts. Mol. Cancer Ther. 2010; 9:1019-1027. [PubMed: 20354120] 
37. Sakamoto J, Kojima H, Kato J, Hamashima H, Suzuki H. Organ-specific expression of the intestinal epithelium-related antigen A33, a cell surface target for antibody-based imaging and treatment in gastrointestinal cancer. Cancer Chemother. Pharmacol. 2000; 46(Suppl):S27-S32. [PubMed: 10950144]

38. Sakamoto J, Oriuchi N, Mochiki E, Asao T, Scott AM, Hoffman EW, Jungbluth AA, Matsui T, Lee FT, Papenfuss A, et al. A phase I radioimmunolocalization trial of humanized monoclonal antibody huA33 in patients with gastric carcinoma. Cancer Sci. 2006; 97:1248-1254. [PubMed: 17034367]

39. Orcutt KD, Rhoden JJ, Ruiz-Yi B, Frangioni JV, Wittrup KD. Effect of small-molecule-binding affinity on tumor uptake in vivo: a systematic study using a pretargeted bispecific antibody. Mol. Cancer Ther. 2012; 11:1365-1372. [PubMed: 22491799]

40. Welt S, Scott AM, Divgi CR, Kemeny NE, Finn RD, Daghighian F, Germain JS, Richards EC, Larson SM, Old LJ. Phase I/II study of iodine 125-labeled monoclonal antibody A33 in patients with advanced colon cancer. J. Clin. Oncol. 1996; 14:1787-1797. [PubMed: 8656247]

41. Scott AM, Lee FT, Jones R, Hopkins W, MacGregor D, Cebon JS, Hannah A, Chong GUP, Papenfuss A, et al. A phase I trial of humanized monoclonal antibody A33 in patients with colorectal carcinoma: biodistribution, pharmacokinetics, and quantitative tumor uptake. Clin. Cancer Res. 2005; 11:4810-4817. [PubMed: 16000578]

42. Carrasquillo JA, Pandit-Taskar N, O’Donoghue JA, Humm JL, Zanzonico P, Smith-Jones PM, Divgi CR, Pryma DA, Ruan S, Kemeny NE, et al. 124)I-huA33 antibody PET of colorectal cancer. J. Nucl. Med. 2011; 52:1173-1180. [PubMed: 21764796]

43. Vocadlo DJ, Hang HC, Kim EJ, Hanover JA, Bertozzi CR. A chemical approach for identifying OGlcNAc-modified proteins in cells. Proc. Natl. Acad. Sci. U. S. A. 2003; 100:9116-9121. [PubMed: 12874386]

44. Clark PM, Dweck JF, Mason DE, Hart CR, Buck SB, Peters EC, Agnew BJ, Hsieh-Wilson LC. Direct in-gel fluorescence detection and cellular imaging of O-GlcNAc-modified proteins. J. Am. Chem. Soc. 2008; 130:11576-11577. [PubMed: 18683930]

45. Boeggeman E, Ramakrishnan B, Kilgore C, Khidekel N, Hsieh-Wilson LC, Simpson JT, Qasba PK. Direct identification of nonreducing GlcNAc residues on N-glycans of glycoproteins using a novel chemoenzymatic method. Bioconjugate Chem. 2007; 18:806-814.

46. Boeggeman E, Ramakrishnan B, Pasek M, Manzoni M, Puri A, Loomis KH, Waybright TJ, Qasba PK. Site specific conjugation of fluoroprobes to the remodeled Fc N-glycans of monoclonal antibodies using mutant glycosyltransferases: application for cell surface antigen detection. Bioconjugate Chem. 2009; 20:1228-1236.

47. Qasba PK, Boeggeman E, Ramakrishnan B. Site-specific linking of biomolecules via glycan residues using glycosyltransferases. Biotechnol. Prog. 2008; 24:520-526. [PubMed: 18426242]

48. Kolb HC, Finn MG, Sharpless KB. Click Chemistry: Diverse Chemical Function from a Few Good Reactions. Angew. Chem., Int. Ed. 2001; 40:2004-2021.

49. Ramakrishnan B, Qasba PK. Structure-based design of beta 1,4-galactosyltransferase I (beta 4Gal$\mathrm{T} 1)$ with equally efficient $\mathrm{N}$-acetylgalactosaminyltransferase activity: point mutation broadens beta 4Gal-T1 donor specificity. J. Biol. Chem. 2002; 277:20833-20839. [PubMed: 11916963] 
A.<smiles>[R7]Cc1ccc(-c2nncnn2)cc1</smiles>
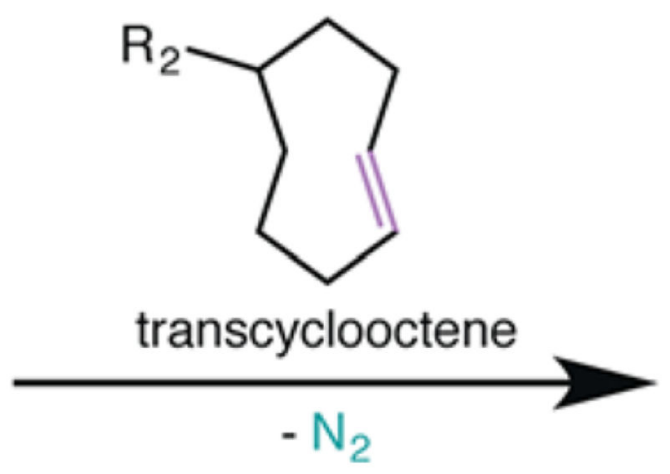

Tetrazine

B.<smiles>[2H]N</smiles><smiles>[R2]C1Cc2ccccc2C#Cc2ccccc21</smiles>

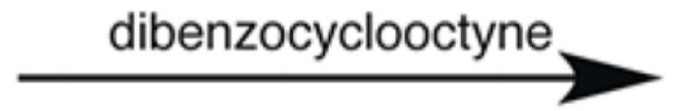

Azide

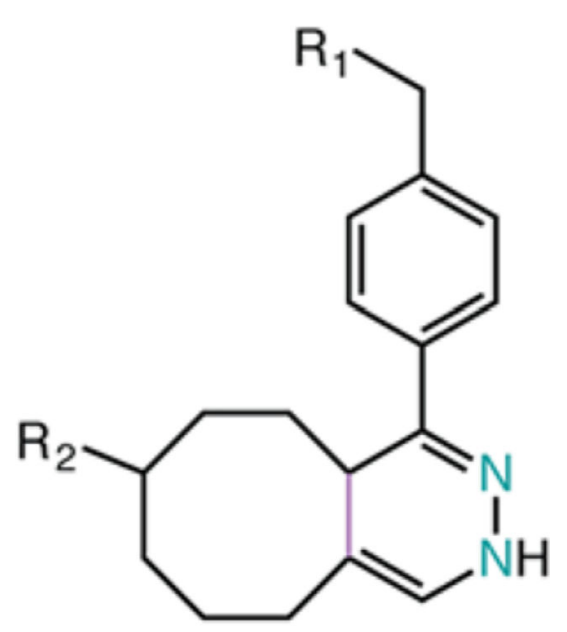<smiles>[R2]C1Cc2ccccc2-c2c(nnn2[R7])-c2ccccc21</smiles>

Figure 1.

Two bioorthogonal reactions employed in this investigation: (A) inverse electron demand Diels-Alder cycloaddition and (B) strain-promoted azide-alkyne cycloaddition. 
A.

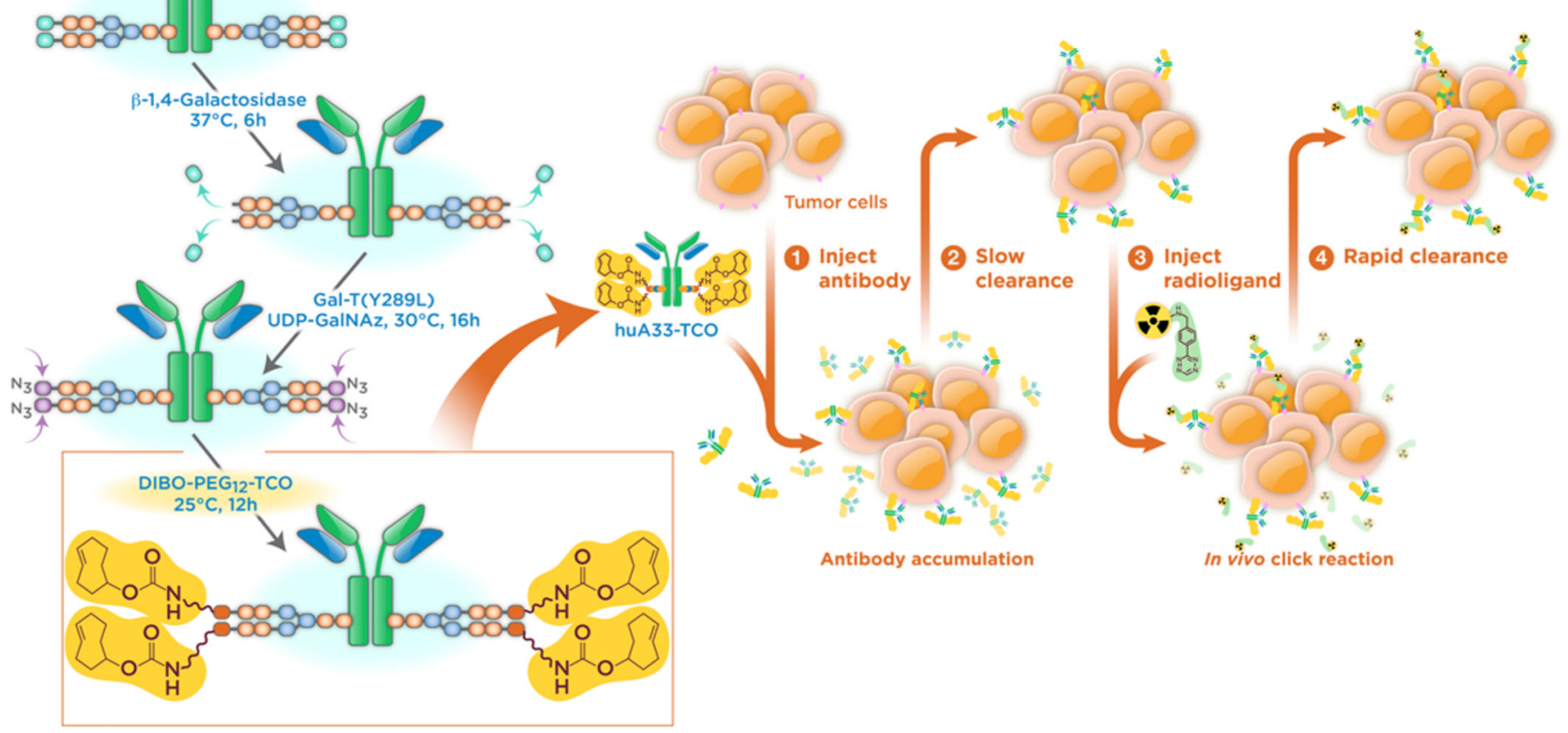

Figure 2.

Schematics of (A) the chemoenzymatic synthesis of ss huA33-PEG ${ }_{12}-\mathrm{TCO}$ and (B) the bioorthogonal approach to pretargeted PET imaging. 


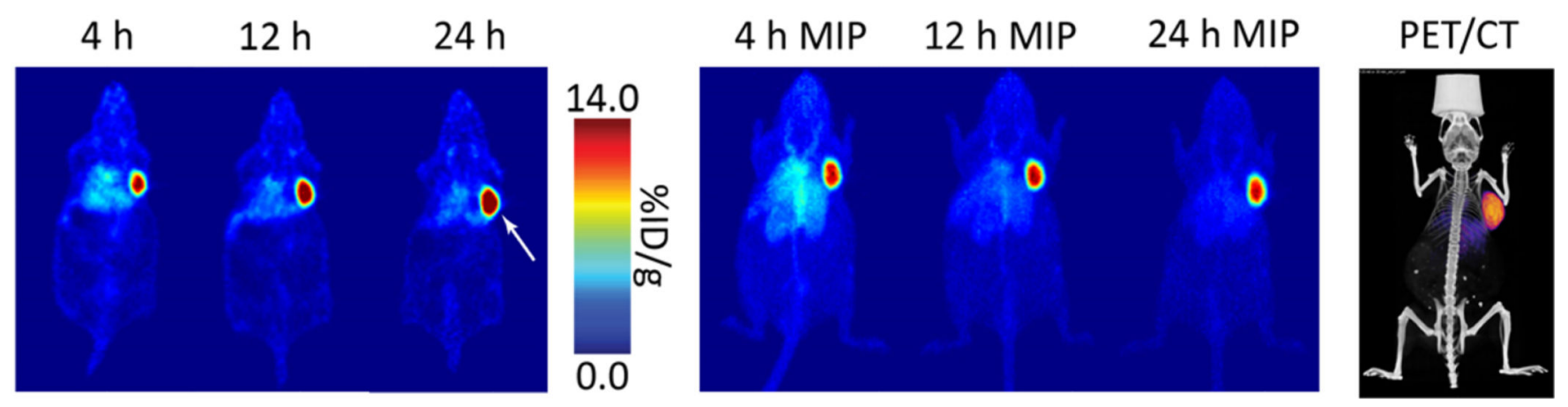

Figure 3.

Pretargeted PET imaging using ss huA33-PEG 12 -TCO (100 $\mu \mathrm{g} ; 0.66 \mathrm{nmol}),{ }^{64} \mathrm{Cu}-\mathrm{Tz}-\mathrm{SarAr}$ (300-350 $\mu \mathrm{Ci}$; 11.1-13.0 MBq; 0.66-0.77 nmol), and a $24 \mathrm{~h}$ accumulation interval. (Left) Coronal slices demonstrating high uptake in the tumor (white arrow); (center) maximum intensityprojections (MIP) showing clearance from background organs; (right) a coregistered PET/CT scan obtained $24 \mathrm{~h}$ after the administration of the radioligand. 


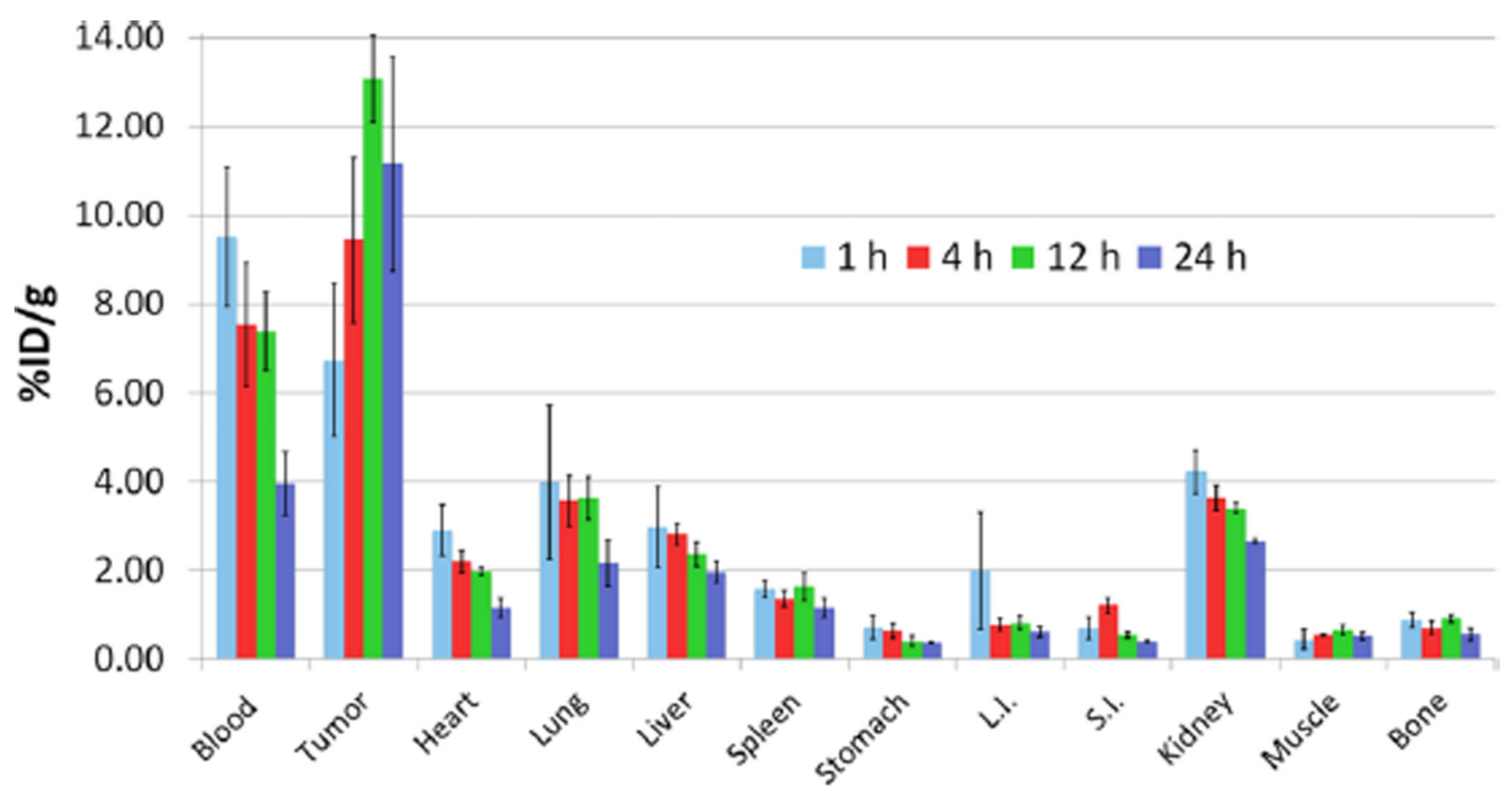

Tissue

Figure 4.

Biodistribution of pretargeting using ${ }^{\text {ss huA33-PEG }} 12$-TCO $(100 \mu \mathrm{g} ; 0.66 \mathrm{nmol}),{ }^{64} \mathrm{Cu}-\mathrm{Tz}-$ SarAr (300-350 $\mu \mathrm{Ci} ; 11.1-13.0 ; 0.66-0.77 \mathrm{nmol})$, and a $24 \mathrm{~h}$ accumulation interval in athymic nude mice bearing A33 antigen-expressing SW1222 human colorectal carcinoma xenografts. 